\title{
Isolated oculomotor nerve palsy resulting from acute traumatic tentorial subdural hematoma
}

This article was published in the following Dove Press journal:

Open Access Emergency Medicine

31 October 2016

Number of times this article has been viewed

\author{
Victoria Cui' \\ Timur Kouliev² \\ 'Washington University School \\ of Medicine, St Louis, MO, USA; \\ ${ }^{2}$ Emergency Department, Beijing \\ United Family Hospital, Beijing, China
}

Correspondence: Victoria Cui 640I Deer Hollow Lane, Austin, TX 78750, USA

Tel + I 5I2 $28937 \mid 8$

Email cui.victoria@wustl.edu

\begin{abstract}
Acute subdural hematoma (SDH) resulting from head trauma is a potentially lifethreatening condition that requires expedient diagnosis and intervention to ensure optimal patient outcomes. Rapidly expanding or large hematomas, elevated intracranial pressure, and associated complications of brain herniation are associated with high mortality rates and poor recovery of neurological function. However, smaller bleeds (clot thickness $<10 \mathrm{~mm}$ ) or hematomas occurring in infrequent locations, such as the tentorium cerebelli, may be difficult to recognize and patients may present with unusual or subtle signs and symptoms, including isolated cranial nerve palsies. Knowledge of neuroanatomy supported by modern neuroimaging can greatly aid in recognition and diagnosis of such lesions. In this report, we present a case of isolated oculomotor nerve palsy resulting from compressive tentorial SDH following blunt head trauma, review the literature concerning similar cases, and make recommendations regarding the diagnosis of SDH in patients presenting with isolated cranial nerve palsies.
\end{abstract}

Keywords: head injury, oculomotor, palsy, subdural hematoma, trauma, tentorium, cerebral herniation, intracranial hemorrhage

\section{Introduction}

Acute subdural hematoma $(\mathrm{SDH})$ is a potentially life-threatening consequence of head trauma which must be recognized early so that appropriate interventions can be initiated. ${ }^{1}$ In patients whose SDH requires surgery, mortality estimates range from $40 \%$ to $65 \%$, and these rates are higher still in certain high-risk populations. ${ }^{2,3}$ Mortality rates as low as $16 \%-17 \%$, however, have been reported for SDH treated at a Level I trauma center or in which the interval between coma onset and surgical decompression was less than 2 hours. ${ }^{4,5}$ In addition to decreasing mortality risk, early diagnosis and effective treatment can determine the degree of central nervous system function recovery achieved by patients with neurological deficits related to their SDH. ${ }^{6,7}$

Most commonly, the source of bleeding in acute traumatic SDH is the tearing of the bridging veins of the dura, which drain into the dural sinuses. Depending on size and location, this pooling of blood and ensuing clot in the subdural space can produce both localized and widespread effects on the brain. An expanding SDH can cause increased intracranial pressure (ICP), with accompanying general symptoms of headache, vomiting, ataxia, and deterioration of consciousness, the last of which may occur as a sign of worsened ICP and cerebral herniation. ${ }^{8} \mathrm{SDH}$ occurrence in specific locations may present with additional findings. In tentorial SDH, the hematoma originates on the surface of the tentorium cerebelli, an extension of dura mater separating 
the cerebellum and occipital lobes, and may compress the cerebral hemisphere or the cerebellum. ${ }^{9}$ Due to the long and winding course of cranial nerves along the tentorial edge, tentorial SDH may also present with abnormalities of the third, fourth, or sixth cranial nerves. ${ }^{10}$ The characteristic pattern of neurological deficits specific to disruption of each cranial nerve is termed a "palsy" and can serve as a diagnostic indicator of the precipitating injury or lesion. Palsies may be classified as "partial" or "complete", depending on whether certain or all findings are present. ${ }^{11-13}$

In oculomotor nerve palsy (ONP), it is the third cranial nerve (CNIII) that is affected. In addition to trauma, other leading causes of ONP include vascular disease, posterior circulation aneurysms, inflammatory injury, and neoplasms. ${ }^{8}$ Patients with ONP may first notice diplopia and ptosis, and upon examination may be found to have anisocoria, pupillary dilation, loss of pupillary reflex, light sensitivity, and weakness of the extraocular muscles resulting in intorsion of the affected eye. ${ }^{14}$ In cases that can be attributed to trauma, isolated ONP is relatively uncommon, as patients usually present with additional neurological deficits. ${ }^{15}$ The incidence of isolated ONP is not known, as no such cases of traumatic isolated ONP have been reported in retrospective epidemiologic studies on cranial NPs, and current knowledge is limited to separate case reports. ${ }^{15-17}$

In this report, we present a case of isolated, ipsilateral ONP resulting from a compressive tentorial SDH following blunt head trauma. This unusual presentation of ONP is followed by a review of existing literature concerning the locations of SDH and the pathophysiology of traumatic gaze palsies, along with a survey of related cases. Imaging technology has progressed rapidly in recent decades, and we conclude with recommendations for clinicians should they encounter patients with symptoms consistent with isolated gaze palsies in the contemporary acute care setting.

Beijing United Family Hospital does not require Ethics Committee approval and/or patient consent in publications where identifying information is not present.

\section{Case report}

A 54-year-old male presented to the emergency department complaining of right frontal headache and diplopia. The patient reported blunt head trauma due to assault, whereby his head had been struck against a concrete wall an hour prior to arrival. He had no loss of consciousness and no vomiting. The patient indicated that he was a nonsmoker, had no past medical conditions, and was not taking anticoagulants or antiplatelet agents. On initial assessment, he was fully awake and appropriate. Physical exam revealed a dilated right pupil, ptosis, and right-gaze palsy with intorsion and paralysis of adduction, elevation, and depression, symptoms consistent with partial CNII dysfunction. Noncontrast computed tomography (CT) of the head was subsequently ordered, and indicated a right tentorial SDH measuring 0.9 $\mathrm{cm}$ in thickness, with absence of mass effect on the cerebral hemisphere (Figures 1 and 2). The patient was expeditiously transferred from China to a tertiary care facility with a dedicated neurosurgical service in Singapore, where he underwent

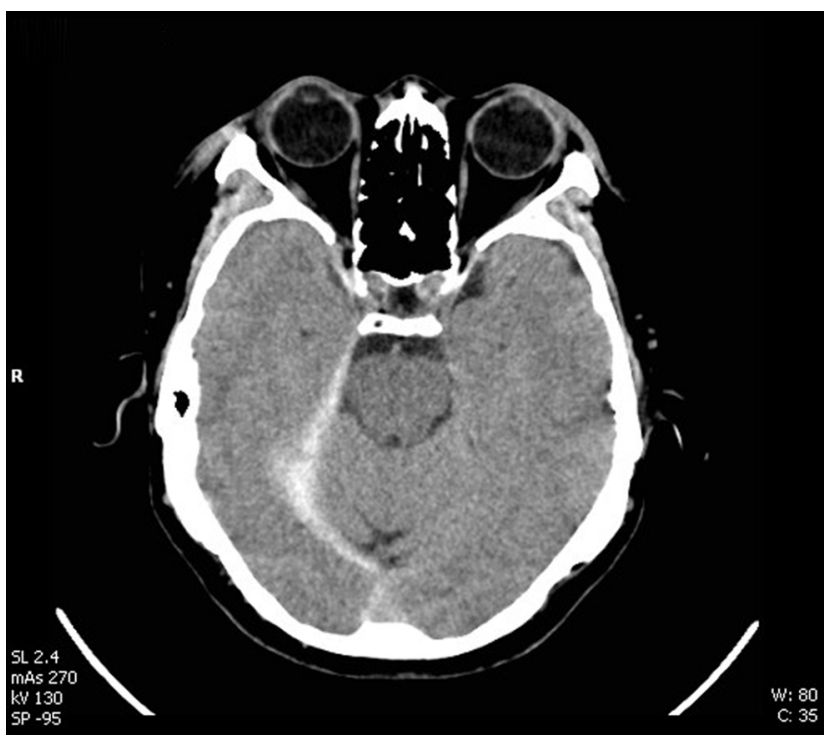

Figure I Noncontrast CT scan of the head prior to surgical evacuation (superior section).

Notes: CT demonstrates a hyperdense area, identified as a right tentorial subdural hematoma measuring $0.9 \mathrm{~cm}$ in thickness, with absence of mass effect on the cerebral hemisphere.

Abbreviation: CT, computed tomography.

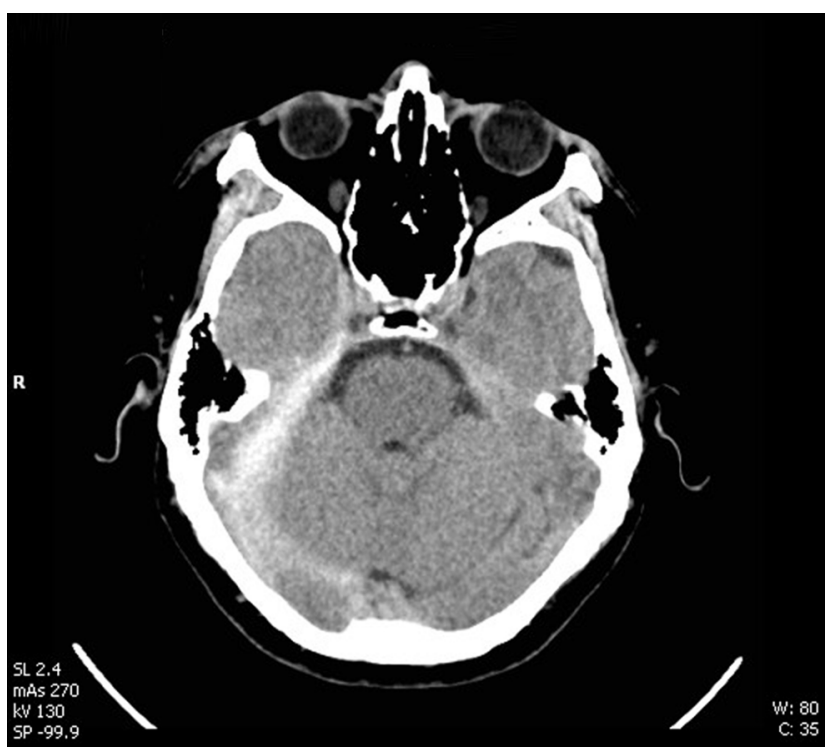

Figure 2 Noncontrast CT scan of the head prior to surgical evacuation (inferior section).

Abbreviation: CT, computed tomography. 
craniotomy for evacuation of the SDH. Due to Singapore's privacy laws, the patient was subsequently lost to follow-up.

\section{Discussion}

Third, fourth, and sixth nerve palsies are commonly used as clinical indicators of neurological injury, which can induce a variety of mechanical stresses or insults to nerve fibers along their paths. Injuries to cranial nerves can be direct or indirect, with possible causes of direct injury to the CNIII leading to palsy, including rootlet avulsion, distal fascicular damage, stretching of the nerve (including the parasellar segment), and a decrease in blood supply. ${ }^{14,18,19}$ In trauma, hemorrhagic injury to the brain stem may result in damage to the nuclear or fascicular portions of the nerve, while more peripheral segments of the nerve can be damaged by fractures or intracranial hemorrhage that extend through the cavernous sinus or superior orbital fissure. ${ }^{18,19}$ Indirect causes of ONP include increased ICP or local compression and displacement of the nerve along its path as the result of aneurysms, traumatic hematomas, and other lesions. ${ }^{20}$ These lesions may result in ONP by causing uncal herniation, vascular damage, brain stem central lesions, stretching of CNIII over the clivus of the sphenoid bone, or compression on CNIII as it passes between the posterior cerebral artery and superior cerebellar artery. $8,11,13,14,21,22$

When associated with traumatic injury, the presentation of ONP is concerning for ipsilateral uncal herniation from mass effect, where the entirety of the cerebral hemisphere is compressed inferiorly onto the edge of the tentorium cerebelli. ${ }^{23}$ The mental status of patients with transtentorial downward brain herniation is generally severely altered, and patients may present with coma and respiratory failure. ${ }^{24}$ The severity and specific combination of symptoms in each patient presenting with ONP reflect the underlying cause of injury, location of damage, and degree of disruption to the nerve. ${ }^{14}$ In the case presented here, ONP following blunt head trauma is unusual in that the ONP resulting from acute SDH is isolated, rather than accompanied by additional neurological deficits. A survey of the literature indicates that the last time such cases were described was in 1953, by Clark and Gooddy. ${ }^{25}$ Since then, knowledge of cranial lesions, standards of care, and the imaging techniques available to clinicians has progressed dramatically.

Similar to the case presented in this report, Clark and Gooddy described two patients in whom "the only definite abnormal sign at the onset was a third cranial NP on the side of the lesion". ${ }^{25}$ Both of these patients also had a recent history of blunt head trauma, and each complained of severe headache. A large SDH was discovered only postmortem in the first patient, with right uncal herniation and notching of the left crus from pressing against the edge of the tentorium. By the time of death, the SDH had progressed to cause significant compression of the right cerebral hemisphere. ${ }^{25}$ The second patient treated by Clark and Gooddy was initially diagnosed with sudden dilation of an aneurysm of a basal vessel. Upon deterioration in status, angiography performed 2 weeks after initial hospital admission indicated subdural collection of blood at the tentorium in the region supplied by the sylvian vessels. ${ }^{25}$ In both cases, subdural bleeding had not been suspected, due to the isolated involvement of CNIII. While the prognosis of these patients cannot be definitively predicted in the context of technology and treatment options available today, these cases speak to the importance of considering and correctly diagnosing serious cerebral lesions from limited clinical data.

Neuroimaging plays an important role in identifying potentially life-threatening lesions, and in the decades since Clark and Gooddy first published their observations, radiological techniques have improved in both technology and availability to clinicians. Given the location of the SDH identified on head CT in this case report, we infer that the acute SDH specifically compressed the CNIII along the edge of the tentorium, leading to intact mental status and symptoms of ipsilateral, partial ONP. After synapsing at the ciliary ganglion, parasympathetic pupillomotor fibers run along the exterior of CNIII. ${ }^{26}$ These are joined by postganglionic sympathetic fibers from the superior cervical ganglion, which innervate the superior tarsal muscle. Compression or other mechanical disturbance to these autonomic fibers is implicated in causing papillary dilation and ptosis, with loss of pupillary muscle contraction and tonic contraction of the superior tarsal muscle, respectively. Further compression of the CNIII at the same site is likely to have disrupted somatic innervation to the medial rectus, superior rectus, inferior rectus, and inferior oblique. These effects caused the patient to experience partial right-gaze palsy with exotropia, with the eye in a "down and out" orientation, manifesting as strabismus and diplopia. In some cases of ONP, there may be "pupil sparing", in which the pupils are not involved. The relative resistance of parasympathetic fibers to ischemia compared to sympathetic and somatic nerve fibers has been proposed as a mechanism for this phenomenon. ${ }^{27,28}$ Pupil-sparing effects were not observed in our patient. We noted that the patient was not known to have any risk factors of SDH or ischemia to cranial nerve fibers, which include smoking history, chronic alcoholism, previous traumatic brain injury, arachnoid cysts, coagulopathy, anticoagulant therapy, cardiovascular disease, thrombocytopenia, and diabetes mellitus. ${ }^{29-31}$ Further information regarding the patient's prognosis following surgical evacuation of SDH 
was not available, due to regional differences in privacy laws, and represents a limitation to this study.

Anatomical variation influences how neurological symptoms may manifest in patients with cerebral lesions or trauma. Five patients have previously been described with lateralized, isolated ONP without pupillary sparing caused by bilateral chronic SDH (CSDH). ${ }^{32-34}$ In one case where CSDH was found to be of similar size bilaterally, the authors proposed that variation in the path of CNIII along the tentorium and uncus and asymmetry of the perimesencephalic cistern could have caused only the right CNIII to be affected. ${ }^{32}$ Slight cerebral peduncle displacement may also result in isolated ONP specific to one side, due to Kernohan's notch phenomena. ${ }^{34}$ In patients with bilateral CSDH where one subdural collection was seen to be larger on CT or during surgical intervention, ipsilateral isolated ONP has also been observed. ${ }^{34}$ Preexisting defects may also predispose certain individuals toward presenting with isolated ONP following even mild trauma, with one patient reported as having a calcified posterior petroclinoid ligament that impinged upon CNIII with impact to the head..$^{35}$

In addition to acute and chronic SDH, other types of intracranial bleeds can also be implicated in isolated ONP. Epidural hematoma in the left temporal region has been identified in an adult patient exhibiting complete ONP but no other neurological symptoms after a fall, and a similar case has been reported in a young adolescent patient. ${ }^{36,37}$ ONP with deficits in both eyes accompanied by anisocoria has also been described, with magnetic resonance imaging (MRI) indicating a focal midbrain hematoma involving the part of the CNIII fascicle that runs between the left cerebral peduncle and red nucleus. ${ }^{38}$ Edema around the hemorrhage was suggested as directly affecting the oculomotor nucleus or Perlia nucleus, manifesting as ONP symptoms. ${ }^{38}$

Standard neuroimaging cannot always provide structural correlation with clinical symptoms. Several cases of isolated ONP have been reported following mild closed head trauma, with no abnormalities seen on brain CT, brain MRI, MR angiography, or orbital CT. ${ }^{15,20,39,40}$ No clear mechanism of damage could be attributed to the observed deficits, because posttraumatic structural changes were not detected by noninvasive imaging. Localized neurosurgical intervention was not indicated, and these patients were reported as experiencing only partial recovery of CNIII function over the long term. ${ }^{15,20,39,40}$ Nonmechanical causes of nerve injury, such as proximal disturbances of blood supply or changes in the biochemical environment arising from head trauma, have been suggested in these cases. ${ }^{7}$

While relatively uncommon, cases of isolated ONP should be viewed by clinicians as suggestive of additional neurological injury and in need of further workup, especially if head trauma is reported or suspected. The differential diagnosis should include acute SDH and other types of intracranial hemorrhage, and we recommend that patients undergo $\mathrm{CT} / \mathrm{CT}$ angiography/MRI to rule out or confirm these possibilities and to determine appropriate interventions.

\section{Conclusion}

Clinical perspectives and standards of care have changed dramatically since Clark and Gooddy noted that "in all but the elderly patient, an uncomplicated [SDH] is a benign lesion if recognized and treated promptly". ${ }^{25}$ SDH and other intracranial bleeds can be life-threatening or cause significant, long-lasting functional deficits if left unrecognized, so it is vital that clinicians be aware that these conditions can manifest with diverse presentations. This unusual case of acute traumatic tentorial SDH causing isolated ONP suggests the need for increased clinician awareness of the different ways that cerebrovascular lesions may affect cranial nerves and manifest in patients. We recommend CT/CT angiography neuroimaging in all patients who present with isolated ONP in order to ensure accurate diagnosis and timely intervention.

\section{Disclosure}

The authors report no conflicts of interest in this work.

\section{References}

1. Bullock MR, Chesnut R, Ghajar J, et al. Surgical management of acute subdural hematomas. Neurosurgery. 2006;58(3 Suppl):S16-S24; discussion Si-Siv.

2. Lukasiewicz AM, Grant RA, Basques BA, Webb ML, Samuel AM, Grauer JN. Patient factors associated with 30-day morbidity, mortality, and length of stay after surgery for subdural hematoma: a study of the American College of Surgeons National Surgical Quality Improvement Program. J Neurosurg. 2016;124(3):760-766.

3. Leitgeb J, Mauritz W, Brazinova A, et al. Outcome after severe brain trauma due to acute subdural hematoma. J Neurosurg. 2012;117(2): 324-333.

4. Ryan CG, Thompson RE, Temkin NR, Crane PK, Ellenbogen RG, Elmore JG. Acute traumatic subdural hematoma: current mortality and functional outcomes in adult patients at a level I trauma center. J Trauma Acute Care Surg. 2012;73(5):1348-1354.

5. Haselsberger K, Pucher R, Auer LM. Prognosis after acute subdural or epidural haemorrhage. Acta Neurochir (Wien). 1988;90(3-4): 111-116.

6. Weimer JM, Gordon E, Frontera JA. Predictors of functional outcome after subdural hematoma: a prospective study. Neurocrit Care. Epub 2016 May 26.

7. Oh CH, Shim YS, Yoon SH, Hyun D, Park H, Kim E. Early decompression of acute subdural hematoma for postoperative neurological improvement: a single center retrospective review of 10 years. Korean J Neurotrauma. 2016;12(1):11-17.

8. Akagi T, Miyamoto K, Kashii S, Yoshimura N. Cause and prognosis of neurologically isolated third, fourth, or sixth cranial nerve dysfunction in cases of oculomotor palsy. Jpn J Ophthalmol. 2008;52(1):32-35. 
9. Matsumoto K, Houri T, Yamaki T, Ueda S. Traumatic acute subdural hematoma localized on the superior surface of the tentorium cerebelli: two case reports. Neurol Med Chir (Tokyo). 1996;36(6):377-379.

10. Chu K, Kang DW, Kim DE, Roh JK. Cerebral venous thrombosis associated with tentorial subdural hematoma during oxymetholone therapy. J Neurol Sci. 2001;185(1):27-30.

11. Schumacher-Feero LA, Yoo KW, Solari FM, Biglan AW. Third cranial nerve palsy in children. Am J Ophthalmol. 1999;128(2):216-221.

12. Flanders M, Hasan J, Al-Mujaini A. Partial third cranial nerve palsy: clinical characteristics and surgical management. Can J Ophthalmol. 2012;47(3):321-325.

13. Berlit P. Isolated and combined pareses of cranial nerves III, IV and VI: a retrospective study of 412 patients. J Neurol Sci. 1991;103(1):10-15.

14. Sadagopan KA, Wasserman BN. Managing the patient with oculomotor nerve palsy. Curr Opin Ophthalmol. 2013;24(5):438-447.

15. Muthu P, Pritty P. Mild head injury with isolated third nerve palsy. Emerg Med J. 2001;18(4):310-311.

16. Memon MY, Paine KW. Direct injury of the oculomotor nerve in craniocerebral trauma. J Neurosurg. 1971;35(4):461-464.

17. Tiffin PA, MacEwen CJ, Craig EA, Clayton G. Acquired palsy of the oculomotor, trochlear and abducens nerves. Eye (Lond). 1996;10(3):377-384.

18. Blake PY, Mark AS, Kattah J, Kolsky M. MR of oculomotor nerve palsy. Am J Neuroradiol. 1995;16(8):1665-1672.

19. Heinz J. Cranial nerve avulsion and other neural injuries. Med J Aust. 1969;2(25):1246-1249.

20. Najafi MR, Mehrbod N. Isolated third nerve palsy from mild closed head trauma. Arch Iran Med. 2012;15(9):583-584.

21. Jo YS, Kim SK, Kim DH, Kim JH, Na SJ. Complete oculomotor nerve palsy caused by direct compression of the posterior cerebral artery. J Stroke Cerebrovasc Dis. 2015;24(7):e189-e190.

22. Otsuka SI, Yamazoe N, Kikuta K, Kunieda T. [Study on cases with primary traumatic oculomotor nerve palsy]. Nihon Geka Hokan. 1994;63(3):87-90. Japanese.

23. Feldmann E, Gandy SE, Becker R, et al. MRI demonstrates descending transtentorial herniation. Neurology. 1988;38(5):697-701.

24. Bruner DI, Jamros C, Cogar W. Subdural hematoma presenting as recurrent syncope. J Emerg Med. 2015;49(3):e65-e68.

25. Clark ES, Gooddy W. Ipsilateral third cranial nerve palsy as a presenting sign in acute subdural haematoma. Brain. 1953;(76):266-278.
26. Sunderland S. Mechanism responsible for changes in the pupil unaccompanied by disturbances of extra-ocular muscle function. $\mathrm{Br} J$ Ophthalmol. 1952;36(11):638-44.

27. Nadeau SE, Trobe JD. Pupil sparing in oculomotor palsy: a brief review. Ann Neurol. 1983;13(2):143-148.

28. Kaido T, Tanaka Y, Kanemoto Y, Katsuragi Y, Okura H. Traumatic oculomotor nerve palsy. J Clin Neurosci. 2006;13(8):852-855.

29. McGirr A, Berlim MT, Bond DJ, et al. A systematic review and metaanalysis of randomized controlled trials of adjunctive ketamine in electroconvulsive therapy: efficacy and tolerability. J Psychiatr Res. 2015;62:23-30.

30. Doherty DL. Posttraumatic cerebral atrophy as a risk factor for delayed acute subdural hemorrhage. Arch Phys Med Rehabil. 1988;69(7):542-544.

31. Hylek EM, Singer DE. Risk factors for intracranial hemorrhage in outpatients taking warfarin. Ann Intern Med. 1994;120(11):897-902.

32. Phookan G, Cameron M. Bilateral chronic subdural haematoma: an unusual presentation with isolated oculomotor nerve palsy. $J$ Neurol Neurosurg Psychiatry. 1994;57(9):1146.

33. Cameron MM. Chronic subdural haematoma: a review of 114 cases. J Neurol Neurosurg Psychiatry. 1978;41(9):834-839.

34. Corrivetti F, Moschettoni L, Lunardi P. Isolated oculomotor nerve palsy as presenting symptom of bilateral chronic subdural hematomas: two consecutive case report and review of the literature. World Neurosurg. 2016;88:686.e9-e12.

35. Patwardhan MA. Isolated unilateral oculomotor nerve neuropraxia following a trivial fall in a patient with calcified posterior petroclinoid ligament. Asian J Neurosurg. 2015;10(1):56.

36. Diyora B, Kukreja S, Nayak N, Kamble H, Sharma A. Complete third nerve palsy: only presenting sign of extradural hematoma in an awake patient. Oman J Ophthalmol. 2014;7(2):103-104.

37. DiTullio MV. Epidural hematoma with complete third nerve paralysis in an awake patient. Surg Neurol. 1977;7(4):193-194.

38. Mizushima H, Seki T. Midbrain hemorrhage presenting with oculomotor nerve palsy: case report. Surg Neurol. 2002;58(6):417-420.

39. Chen CC, Pai YM, Wang RF, Wang TL, Chong CF. Isolated oculomotor nerve palsy from minor head trauma. Br J Sports Med. 2005;39(8):e34.

40. Erenler AK, Yalçın A, Baydin A. Isolated unilateral oculomotor nerve palsy due to head trauma. Asian J Neurosurg. 2015;10(3):265-267.
Open Access Emergency Medicine

\section{Publish your work in this journal}

The Open Access Emergency Medicine is an international, peerreviewed, open access journal publishing original research, reports, editorials, reviews and commentaries on all aspects of emergency medicine. The manuscript management system is completely online and includes a very quick and fair peer-review system, which is all

\section{Dovepress}

easy to use. Visit http://www.dovepress.com/testimonials.php to read real quotes from published authors. 